\title{
Reversal of dilated cardiomyopathy after glucagonoma excision
}

\author{
Ozan M. Demir, ${ }^{1}$ Stavroula A. Paschou, ${ }^{2}$ Huw Christopher Ellis, ${ }^{1}$ \\ Michael Fitzpatrick, ${ }^{1}$ Andreas S. Kalogeropoulos, ${ }^{1}$ Andrew Davies, ${ }^{3}$ \\ Jeremy Thompson, ${ }^{3}$ Simon W. Davies, ${ }^{1}$ Julia Grapsa ${ }^{1}$
}

${ }^{1}$ Department of Cardiology, Royal Brompton Hospital, Royal Brompton \& Harefield NHS Trust, ${ }^{2}$ Department of
Diabetes, St Bartholomew's Hospital, ${ }^{3}$ Department of General Surgery, Royal Marsden Hospital, London, UK

To the Editor,

The following case presents a new insight into the relationship of glucagonomas with dilated cardiomyopathy which endocrinologists should be aware of.

A 64-year old Caucasian man, born and raised in Zimbabwe, was admitted with acute pulmonary edema and left ventricular failure. He was an ex-smoker and consumed minimal alcohol. He did not have any family history of cardiac disease or any history of childhood or other illnesses. He had a resting ECG in sinus rhythm, left axis deviation and voltage criteria for left ventricular hypertrophy, a QRS duration of $102 \mathrm{msec}$ and a QTc interval of $495 \mathrm{~ms}$. Coronary angiography demonstrated only mild atheroma and myocardial perfusion scan indicated that the left ventricular dysfunction was disproportional to the extent of the atheroma.

Key words: Dilated cardiomyopathy, Glucagon, Glucagonoma

\footnotetext{
Corresponding author:

Dr. Julia Grapsa MD, PhD, Department of Cardiology,

Royal Brompton Hospital, Royal Brompton \& Harefield NHS

Trust, London, UK, Tel.: +44 7447053606,

Fax: +44 2083834392, e-mail: jgrapsa@gmail.com

Received 09-07-2014, Accepted 01-08-2014
}

The patient was fully investigated and was diagnosed with idiopathic dilated cardiomyopathy (left ventricular end-diastolic diameter: $70 \mathrm{~mm}$ and a reduced left ventricular ejection fraction of $30 \%$, raised left ventricular filling pressures, E/E' ratio 18 ). Cardiac magnetic resonance confirmed the diagnosis of dilated cardiomyopathy but failed to demonstrate any late gadolinium enhancement to suggest myocardial fibrosis. The patient was started on Ramipril and Bisoprolol and was discharged. Two years later, the patient developed a troublesome itch. During his investigations, a pancreatic mass was discovered. Abdominal computed tomography with contrast demonstrated an enhancing soft tissue mass within the tail of the pancreas which measured approximately $3.5 \mathrm{~cm}$ and contained two dystrophic areas of calcification. The mass was enhanced compared to the surrounding parenchyma. There were no metastases (Figure 1). Glucagon was high at $97 \mathrm{pmol} / \mathrm{lt}$ (normal value $<50 \mathrm{pmol} / \mathrm{lt}$ ) and the diagnosis of pancreatic glucagonoma was made. The patient underwent a distal pancreatectomy and histology of the resected mass confirmed a well differentiated neuroendocrine tumor, grade 1, with positive staining for glucagon (T3N1). One month later, his hormonal profile was normal and the patient underwent an echocardiogram as a part of the usual follow-up of his dilated cardiomyopathy. He was found to have a normal left ventricular ejection fraction of $67 \%$ and significantly 


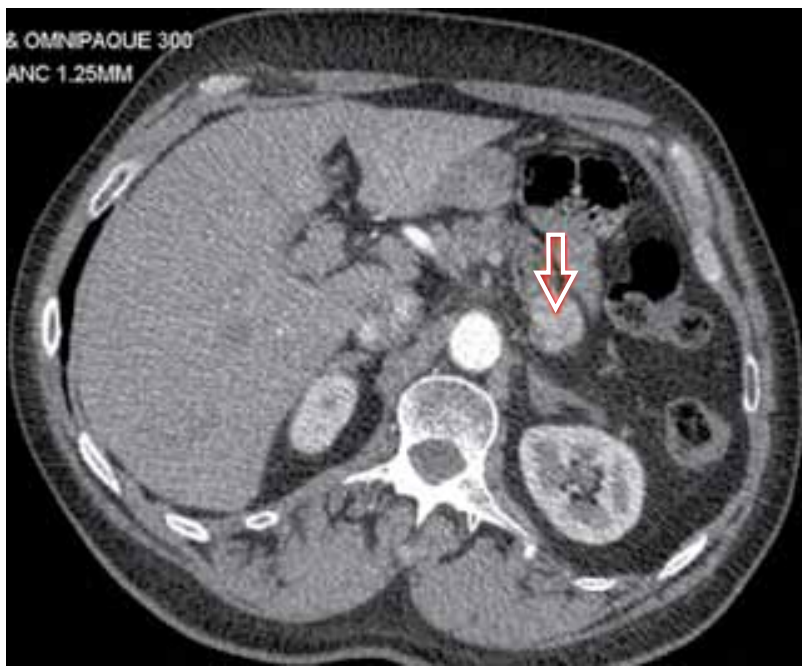

Figure 1.

reduced left ventricular dimensions (end-diastolic: 55 $\mathrm{mm}$, end-systolic: $31 \mathrm{~mm}$ ).

Glucagonoma is a rare neuroendocrine tumor, this being only the second case in the literature, where it has been described as a reversible cause of dilated cardiomyopathy. ${ }^{1}$ Glucagon activates myocardial adenylate cyclase, with an increase in cyclic AMP. This results in increased cardiac demands and may lead to congestive heart failure. Recent research demonstrates that glucagon-like peptide-1 agents, that are broadly used in type 2 diabetes management, increasing insulin and reducing glucagon levels, may be beneficial for patients with chronic heart failure. ${ }^{2,3}$

In conclusion, investigations for the diagnosis of dilated cardiomyopathy should not omit a detailed clinical history, while the possibility of an endocrine mass, even though rare, should always be taken into consideration. Furthermore, endocrinologists and surgeons who treat patients with glucagonomas should be aware of such an association.

\section{CONFLICT OF INTEREST}

The authors declare that they have no conflict of interest.

\section{REFERENCES}

1. Chang-Chretien K, Chew JT, Judge DP, 2004 Reversible dilated cardiomyopathy associated with glucagonoma. Heart 90: e44.

2. Dokken BB, Piermarini CV, Teachey MK, et al 2013 Glucagon-like peptide-1 preserves coronary microvascular endothelial function after cardiac arrest and resuscitation: potential antioxidant effects. Am J Physiol Heart Circ Physiol 304: H538-546.

3. Sokos GG, Nikolaidis LA, Mankad S, Elahi D, Shannon RP, 2006 Glucagon-like peptide-1 infusion improves left ventricular ejection fraction and functional status in patients with chronic heart failure. J Card Fail 12: 694-699. 